\title{
基于手性负离子置换策略的异喹啉不对称转移氢化研究
}

\author{
时䂞 $* a, b \quad$ 姬悦 ${ }^{a}$ 黄文学 ${ }^{a}$ 周永贵*,a \\ $\left({ }^{a}\right.$ 中国科学院大连化学物理研究所 大连 116023) \\ $\left({ }^{b}\right.$ 大连理工大学精细化工国家重点实验室 大连 116024)
}

\begin{abstract}
摘要 报道了一种手性负离子置换策略, 并将其成功应用于异喹啉化合物的不对称转移氢化反应. 以手性磷酸作为催 化剂, 氯代甲酸酯作为底物活化试剂, Hantzsch 酯为有机负氢供体的条件下, 该反应能以优良收率得到手性 1-取代-1,2二氢异喹啉产物, ee 值最高为 $79 \%$. 这是首次实现异喹啉化合物不对称转移氢化.
\end{abstract}

关键词 手性磷酸; 手性负离子; 转移氢化; 异哇啉

\section{Application of Chiral Anion Metathesis Strategy in Asymmetric Transfer Hydrogenation of Isoquinolines}

\author{
Shi, Lei* ${ }^{*, a} \quad \mathrm{Ji}_{\text {, Yue }}{ }^{a} \quad$ Huang, Wenxue $^{a} \quad$ Zhou, Yonggui*, $^{, a}$ \\ ( ${ }^{a}$ Dalian Institute of Chemical Physics, Chinese Academy of Sciences, Dalian 116023, China) \\ ( ${ }^{b}$ State Key Laboratory of Fine Chemicals, Dalian University of Technology, Dalian 116024, China)
}

\begin{abstract}
Asymmetric hydrogenation of $N$-hetero aromatics offers a very straightforward and efficient method to obtain the corresponding chiral $N$-hetero cyclic saturated or partially saturated compounds. As one of the most challenging substrates, asymmetric hydrogenation of isoquinolines has met with limited success probably because of lower reactivity and the catalyst deactivation resulted from strong coordination. Considering the prevalence of the chiral 1,2,3,4-tetrahydroisoquinoline motif in natural alkaloids and drug molecules, the development of new catalyst system for asymmetric hydrogenation of isoquinolines is highly desirable and significant. Herein, a novel chiral anion metathesis strategy successfully applied for asymmetric transfer hydrogenation of isoquinolines is reported. $N$-Protected 1 -substituted 1,2-dihydroisoquinolines were obtained with high yield and up to $79 \%$ ee in the presence of Hantzsch ester and chloroformate using chiral phosphoric acid as catalyst. The phosphate salt and the activated $N$-acyl isoquinolinium chloride undergo anion metathesis to form chiral contact ion pair, which leads to a highly enantioselective transfer hydrogenation of isoquinolines. After systematically investigating the effects of activating reagent, solvent, base, hydride donor and catalyst on this transfer hydrogenation reaction, the best result was achieved under the optimized condition as follows: $5 \mathrm{~mol} \%$ H8-BINOL-derived chiral phosphoric acid as catalyst, 1.2 equivalent 2,2,2-trichloroethyl chloroformate as activator, 1.5 equivalent dimethyl 2,6-diethyl-1,4-dihydropyridine-3,5dicarboxylate as hydride donor, 1.5 equivalent sodium carbonate as base and cyclohexane as solvent. The reaction is tolerant toward a broad range of aryl or alkyl 1-substituted isoquinoline substrates. This methodology represents one of the rare examples of asymmetric hydrogenation of this challenging substrate. The utilizing of chiral anion metathesis strategy could enable chiral phosphoric acid to catalyze more asymmetric transformation process and further researching is ongoing in our laboratory.

Keywords chiral phosphoric acid; chiral anion metathesis; transfer hydrogenation; isoquinolines
\end{abstract}

\section{1 引言}

作为一类重要的有机小分子催化剂, 轴手性联萗二 酚衍生的手性磷酸近些年来异军突起. 从 2004 年由 Akiyama $^{[1]}$ 和 Tereda ${ }^{[2]}$ 首次报道以来, 手性磷酸已经成功 地应用于很多不对称反应，例如 Mannich 反应 ${ }^{[1,2]}$ 、 Streck 反应 ${ }^{[3]}$ 、环加成反应 ${ }^{[4 \sim 8]}$ 、亚胺的还原 ${ }^{[9 \sim 15]}$ 、 Friedel-Crafts ${ }^{[16 ~ 20]} 反$ 应、Baeyer-Villiger 氧化 ${ }^{[2]}$ 等, 并且 获得了非常优异的催化活性和对映选择性, 迅速成长为
一类优势催化剂. 手性磷酸属于酸性较强的 Brønsted 酸，催化原理主要是酸催化机制，提供质子来活化亲电 底物并与之通过氢键作用，从而控制反应的手性传递. 同时, $\mathrm{P}=\mathrm{O}$ 又可以提供孤对电子，作为 Lewis 碱性位点， 这样手性磷酸可以同时活化亲电和亲核底物, 具有双功 能催化剂的特点. 最近，一些基于手性磷酸负离子的新 反应不断涌现, 甚至跨越了有机小分子催化的界限, 迸 发出更加蓬勃的生命力. 手性磷酸催化剂与金属催化剂 协同使用，在金属催化的反应中也能发挥独特的作

*E-mail: shileichem@dicp.ac.cn; ygzhou@dicp.ac.cn

Received May 19, 2014; published June 17, 2014

Supporting information for this article is available free of charge via the Internet at http://sioc-journal.cn.

Project supported by the National Natural Science Foundation of China (No. 21202162) and the State Key Laboratory of Fine Chemicals (No. KF1110).

项目受国家自然科学基金青年科学基金项目(No. 21202162)和大连理工大学精细化工国家重点实验室开放课题基金(No. KF1110)资助. 
用 ${ }^{[22 ~ 27]}$. 在这些反应中, 手性磷酸负离子可以与活性中 间体形成手性离子对，为化学反应过程提供良好的手性 环境. List 等 ${ }^{[28]}$ 在此基础上提出了不对称反离子导向催 化(Asymmetric Counteranion-Directed Catalysis)的概念. Toste 研究小组 ${ }^{[29]}$ 在手性磷酸骨架结构中引入脂溶性基 团, 成功发展出负离子相转移催化的概念 (Anionic Phase-Transfer Catalysis), 实现了不对称卤化环化 ${ }^{[30]}$ 、不 对称氟化去芳构化 ${ }^{[31]}$ 、手性胺的去外消旋化 ${ }^{[32]}$ 等常规方 法不易实现的反应, 为手性磷酸化学开辟了另一片崭新 的天地. 由此可见, 发展新的反应机制使手性磷酸不再 仅仅局限于 Brønsted 酸催化的范畴, 并适用于更广泛更 多样的化学是非常必要和有意义的.

利用 NAD $(\mathrm{P}) \mathrm{H}$ 的模型化合物 Hantzsch 酯(HEH)作 为有机负氢供体 ${ }^{[33]}$, 手性磷酸可以有效催化氮杂芳香 化合物的不对称转移氢化反应 ${ }^{[34]}$. 该反应条件温和, 操 作简便, 对映选择性高, 是合成手性氮杂环化合物的有 效手段, 也是手性磷酸催化剂的重要应用之一. 金属催 化的芳香化合物不对称氢化反应目前已经获得长足的 发展 ${ }^{[35 ~ 46]}$, 与之相比, 手性磷酸催化的转移氢化由于受 限于酸催化机理本身, 目前仅有喹啉 ${ }^{[34,47]}$ 、喹惡啉 ${ }^{[48]}$ 和 某些特殊结构的吡啶 ${ }^{[49]}$ 等三类芳香化合物可以实现该 类反应. 异喹啉化合物因其还原产物具有较强配位能力 和碱性, 易与金属配位或者与手性磷酸生成稳定的酸碱 加成物从而致使催化剂失活. 所以, 异喹啉的不对称氢 化和转移氢化是该领域中的难点, 成功的例子还不多 见 $^{[50 ~ 54]}$. 而且目前还没有关于异喹啉不对称转移氢化 的研究见诸报道. 手性四氢异喹啉是天然产物和药物分 子中常见的结构(图 1), 因此发展新的策略来解决这一难 题, 实现异喹啉的不对称转移氢化具有重要的研究价值.<smiles>COc1cc2c(cc1OC)C(C)N(C)CC2</smiles>

$(-)$-Carnegine

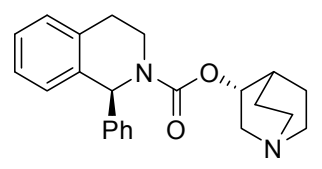

$(+)$-Solifenacin
图 1 含四氢异喹啉骨架的代表性天然生物碱和手性药物

Figure 1 Representative examples of natural alkaloid and drug with chiral tetrahydroisoquinoline skeleton

本文设计了一种手性负离子置换策略(图 2), 通过 采用手性磷酸与酰氯活化的异喹啉底物之间发生离子 复分解反应, 形成手性离子对, 成功实现了异喹啉化合 物的不对称转移氢化反应，该方法可以优异的收率合成 手性 1,2-二氢异喹啉化合物(最高 79\%ee).

\section{2 结果与讨论}

\section{1 转移氢化反应的初步探索}

我们小组在 2006 年采用氯甲酸酯作为芳香底物的 活化试剂, 实现了铱催化异喹啉化合物的不对称氢化反

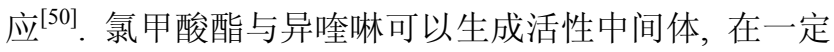
程度上减弱了其芳香性，进而发生氢化反应，得到氮原 子被酰基保护的手性 1,2-二氢异喹啉产物. 此外, 酰基 的引入还有效抑制了底物和氢化产物对金属催化剂的 毒化作用，这也是该方法获得成功的另一个主要原因. 鉴于异喹啉的转移氢化反应也受到类似的困扰，本文尝 试采用这种酰氯活化方式来实现手性磷酸催化的异喹 啉不对称转移氢化反应.

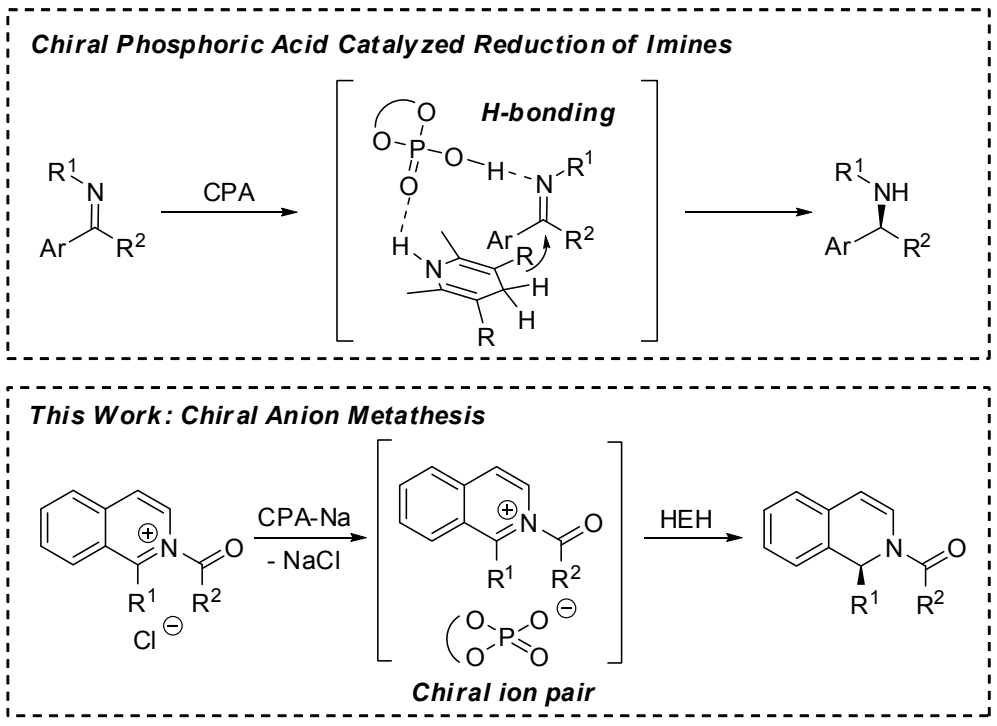

图 2 通过手性负离子置换策略实现异喹啉的不对称转移氢化

Figure 2 Asymmetric biomimetic transfer hydrogenation via chiral anion metathesis strategy 


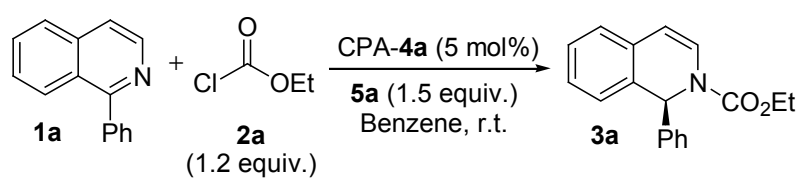

\begin{tabular}{ccc}
$\mathrm{Na}_{2} \mathrm{CO}_{3}$ & ee/\% & Yield/\% \\
\hline 0 & 8 & 40 \\
1.5 equiv. & 18 & 57
\end{tabular}

首先, 采用 1 -苯基异喹啉作为模型底物, $5 \mathrm{~mol} \%$ 手 性磷酸 $\mathbf{4 a}$ 作为催化剂, Hantzsch 酯 $\mathbf{5}$ 作为氢源, 苯作为 反应溶剂, 并加入氯甲酸乙酯为底物活化试剂. 反应在 室温下进行 $48 \mathrm{~h}$, 以 $40 \%$ 的分离收率得到 1,2-二氢异喹 啉产物, 但是产物的对映体过量值仅有 $8 \%$. 而当其它 条件不变, 向反应体系中加入 1.5 equiv. 的 $\mathrm{Na}_{2} \mathrm{CO}_{3}$ 进行 反应时，分离收率提高到 $57 \%$, 对映选择性也明显提高 到 $18 \%$ ee. 无机碱碳酸钠的加入使催化剂转变成磷酸 钠盐形式, 磷酸钠与 $N$-酰基异喹啉中间体之间发生离 子交换, 产生的氯化钠在有机溶剂苯中具有较小的溶解 度, 易从体系中析出, 使复分解反应平衡向右移动. 这
样，手性磷酸负离子就与 $N$-酰基异喹啉中间体形成了 手性离子对, 对异喹啉的不对称氢化过程进行有效的手 性控制. 初步结果显示出这一策略的可行性, 并在此鼓 舞下, 我们对转移氢化的反应条件进行了进一步优化.

\section{2 转移氢化反应的条件优化}

首先对有机溶剂对反应的活性和对映选择性的影 响进行了考察. 反应在苯、甲苯、二氯甲烷、四氢呋喃、 乙醚等溶剂中进行时, 虽然都能获得很好的收率, 但是 对映选择性均不理想 $(e e<20 \%)$. 主要的原因是存在强 烈的背景反应，比如采用苯作为溶剂时，不加入手性磷 酸催化剂也能以 44\%分离收率得到 1,2-二氢异喹啉产 物. 我们发现当反应在正己烷中进行时, 对映选择性可 以得到一定提高(表 1, Entry 1). 推测可能是由于底物和 Hantzsch 酯在饱和烷烃类溶剂中的溶解度都不高, 从 而可以在一定程度上抑制背景反应，有利于反应的对映 选择性控制. 所以，先以正己烷作为反应介质对反应进 行进一步条件优化.

表 1 反应条件的优化

Table 1 Optimization of reaction conditions

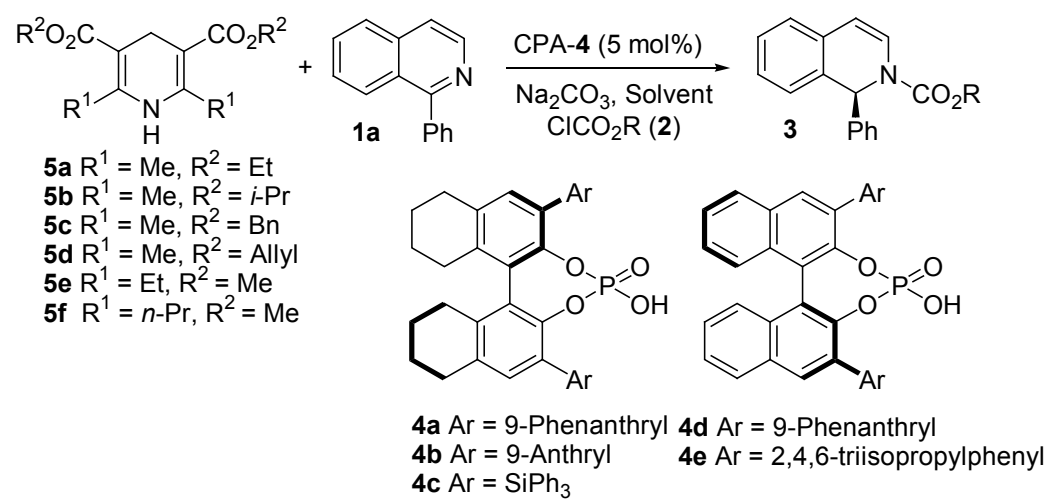

\begin{tabular}{|c|c|c|c|c|c|}
\hline Entry $^{a}$ & $\mathrm{R}\left(\mathrm{ClCO}_{2} \mathrm{R}\right)$ & Solvent & $\mathrm{HEH}$ & Yield/\% & $e e / \%$ \\
\hline 1 & Et & Hexane & $5 a$ & 45 & 30 \\
\hline 2 & $i$-Bu & Hexane & $5 a$ & 58 & 18 \\
\hline 3 & $\mathrm{Bn}$ & Hexane & $5 a$ & 64 & 26 \\
\hline 4 & $\mathrm{CH}_{2} \mathrm{CCl}_{3}$ & Hexane & $5 \mathbf{a}$ & 95 & 50 \\
\hline 5 & $\mathrm{C}(\mathrm{Me})_{2} \mathrm{CCl}_{3}$ & Hexane & $5 a$ & 95 & 17 \\
\hline 6 & $\mathrm{CH}_{2} \mathrm{CCl}_{3}$ & Hexane & $5 \mathbf{b}$ & 79 & 20 \\
\hline 7 & $\mathrm{CH}_{2} \mathrm{CCl}_{3}$ & Hexane & $5 c$ & 95 & 21 \\
\hline 8 & $\mathrm{CH}_{2} \mathrm{CCl}_{3}$ & Hexane & $5 d$ & 95 & 45 \\
\hline 9 & $\mathrm{CH}_{2} \mathrm{CCl}_{3}$ & Hexane & $5 e$ & 84 & 68 \\
\hline 10 & $\mathrm{CH}_{2} \mathrm{CCl}_{3}$ & Hexane & $5 f$ & 95 & 33 \\
\hline $11^{b}$ & $\mathrm{CH}_{2} \mathrm{CCl}_{3}$ & Hexane & $5 e$ & 97 & 22 \\
\hline $12^{c}$ & $\mathrm{CH}_{2} \mathrm{CCl}_{3}$ & Hexane & $5 e$ & 92 & 34 \\
\hline $13^{d}$ & $\mathrm{CH}_{2} \mathrm{CCl}_{3}$ & Hexane & $5 e$ & 95 & 45 \\
\hline 14 & $\mathrm{CH}_{2} \mathrm{CCl}_{3}$ & Pentane & $5 e$ & 97 & 44 \\
\hline 15 & $\mathrm{CH}_{2} \mathrm{CCl}_{3}$ & Cyclopentane & $5 e$ & 95 & 72 \\
\hline 16 & $\mathrm{CH}_{2} \mathrm{CCl}_{3}$ & Cyclohexane & $5 e$ & 95 & 73 \\
\hline $17^{e}$ & $\mathrm{CH}_{2} \mathrm{CCl}_{3}$ & Cyclohexane & $5 e$ & 95 & 61 \\
\hline $18^{f}$ & $\mathrm{CH}_{2} \mathrm{CCl}_{3}$ & Cyclohexane & $5 e$ & 40 & 29 \\
\hline $19^{g}$ & $\mathrm{CH}_{2} \mathrm{CCl}_{3}$ & Cyclohexane & $5 e$ & 92 & -46 \\
\hline $20^{h}$ & $\mathrm{CH}_{2} \mathrm{CCl}_{3}$ & Cyclohexane & $5 e$ & 63 & -18 \\
\hline
\end{tabular}

${ }^{a}$ Reaction condition: $0.1 \mathrm{mmol}$ of 1a, $5.0 \mathrm{~mol} \%$ chiral phosphoric acid 4a, 1.2 equiv. chloroformate, 1.5 equiv. Hantzsch ester, 1.5 equiv. base, $2.0 \mathrm{~mL}$ solvent, room temperature, $24 \mathrm{~h} . \mathrm{Na}_{2} \mathrm{CO}_{3}$ was used as base, unless otherwise noted. ${ }^{b} \mathrm{Li}_{2} \mathrm{CO}_{3} ;{ }^{c} \mathrm{Cs}_{2} \mathrm{CO}_{3} ;{ }^{d}$ DIPEA $;{ }^{e} \mathbf{4 b} ;{ }^{f}(S)-\mathbf{4} \mathbf{c} ;{ }^{g} \mathbf{4 d} ;{ }^{h} \mathbf{4 e}$. 
接着以 $4 \mathrm{a}$ 为催化剂, 对不同的氯甲酸酯进行了笁 选(表 1, Entries 1 5). 当采用位阻较大取代基的酰氯, 如氯甲酸异丁酯、氯甲酸苠酯时, 收率略有提高, 但是 对映选择性有所下降. 当采用含有强吸电取代基的氯甲 酸三氯乙酯( $\mathrm{TrocCl}$ ) 作为底物活化试剂时, 反应活性和 对映选择性均明显改善, 分离收率可提高到 $95 \%$, 产物 的对映体过量值可提高到 50\%(表 1, Entry 4). 采用氯甲 酸 1,1-二甲基-2,2,2-三氯乙酯虽然可以保持很好的反应 收率，但是氢化产物的对映体过量值下降至 $17 \%$. 含较 大位阻取代基的酰氯可能会减弱手性离子对之间的相 互作用, 不利于反应的手性诱导. 所以, 实验结果说明, 对于该反应氯甲酸三氯乙酯是最佳的底物活化试剂.

在转移氢化反应中, Hantzsch 酯的结构会对反应结 果产生重要影响. 采用 $\mathbf{4 a}$ 和 $\mathrm{TrocCl}$, 考察了含不同酯基 和 2,6 位取代基 Hantzsch 酯的影响(表 1, Entries 6 10). 采用 2,6-二甲基 Hantzsch 酯为氢源时, 酯基的位阻增 大, 如异丙酯、苠酯和烯丙酯, 会对反应的对映选择性 产生不利影响. 当 Hantzsch 酯的 2,6 位取代基为乙基, 酯基为位阻较小的甲酯时(HEH 5e), 产物的对映选择性 可以得到进一步提高, $e e$ 值可达到 $68 \%$. 而将 2,6 位取代 基替换为丙基时, 产物的 ee 值却急剧下降, 仅有 $33 \%$. 原因可能是 Hantzsch 酯引入丙基取代后, 在正己烷中 的溶解性变好, 背景反应加重.

随后对碱也作了相关考察(表 1, Entries 11 13), 无 论是无机碱 $\left(\mathrm{Li}_{2} \mathrm{CO}_{3} 、 \mathrm{~K}_{2} \mathrm{CO}_{3} 、 \mathrm{Cs}_{2} \mathrm{CO}_{3}\right.$ 等)还是有机碱类 (DIPEA、 $\mathrm{Et}_{3} \mathrm{~N} 、 \mathrm{DBU}$ 等)都不能得到与 $\mathrm{Na}_{2} \mathrm{CO}_{3}$ 比肩的 结果. 究其原因, 这些碱可能不能很好的促进磷酸盐与 $N$-酰基异喹啉中间体之间的离子复分解反应. 又对饱和 烷烃类溶剂作了更为详细的笁选(表 1, Entries 14 16), 我们发现环状烷烃溶剂更适合该反应, 转移氢化反应在 环己烷中进行, 产物的对映体过量值可提高到 $73 \%$. 最 后对手性磷酸催化剂进行了篎选, 实验结果表明 H8-BINOL 骨架的手性磷酸的选择性明显要好于 BINOL 骨架催化剂. 前者拥有更好的脂溶性能, 形成手 性离子对时, 增加了中间体在烃类反应介质中的溶解 度. 这与 Toste 提出的负离子相转移催化有异曲同工之 处. 手性磷酸的 3,3'-取代基只有是大位阻的芳基取代基 时, 如菲基或者葱基, 才能获得很好的对映选择性. 采 用 3,3'-三苯基硅基的手性磷酸, 收率和 $e e$ 值均不理想. 菲基的大共轭平面可能会产生平面堆积效应从而有利 于反应的对映选择性控制. 催化剂篎选实验的结果表 明, $4 \mathbf{a}$ 为该反应的最佳催化剂.

综上, 最后确定的最佳反应条件为: $5.0 \mathrm{~mol} \%$ 的手 性磷酸 4a, 1.2 equiv. 的氯甲酸三氯乙酯, 1.5 equiv. 的 HEH 5e, 1.5 equiv. 的碳酸钠, 环己烷为反应溶剂.

\section{3 异喹啉转移氢化反应的底物范围}

在确定了最佳反应条件之后, 采用催化剂 $4 \mathrm{a}$ 对底 物范围进行了考察(图 3). 当 1-苯基上含有供电子基团,
如甲基或者甲氧基时，反应均可顺利进行. 对映选择性 普遍比模型底物高，其中 1-(3,5-二甲基)苯基异喹啉底 物在该反应条件下可得到 79\% ee 的手性产物. 取代基 位置对选择性具有重要影响，当 1-苯基的 2 位为甲基取 代时, 只能得到外消旋的氢化产物. 在 1-苯基上引入吸 电子性质的取代基，如 $\mathrm{Cl} 、 \mathrm{~F}$ 时，氢化反应同样可以保 持优异的收率和良好的对映选择性. 6,7-二甲氧基异喹 啉底物在该条件下进行转移氢化反应时，对映选择性会 有较明显的下降. 此外, 1-环戊基异喹啉的转移氢化也 可以得到很好收率和对映选择性，证明该方法具有较好 的底物适用范围. 所得产物 $\mathbf{3 b}$ 的绝对构型由化学相关 法确定为 $S$ 构型(具体参见支持信息). 在所有转移氢化 产物的结构中均保留了 3,4 位双键, 这也为进一步的化 学转化提供了可能的反应位点.<smiles>O=C([O-])N1C=Cc2ccccc2[C@H]1c1ccccc1</smiles><smiles>COc1ccc([C@H]2c3ccccc3C=CN2C(C)([O-])[O-])cc1</smiles><smiles>CC(=O)N1C=Cc2ccccc2C1c1cccc(C)c1</smiles>

(S)-3b $95 \%^{a}, 73 \%$ ee $\quad$ 3c $76 \%, 72 \%$ ee

3d $69 \%, 75 \%$ ee<smiles>Cc1cc(C)cc(C2c3ccccc3C=CN2C(=O)OCc2ccccc2)c1</smiles><smiles>COc1cccc(C2c3ccccc3C=CN2C(C)=O)c1</smiles><smiles>COc1ccc(C2c3ccccc3C=CN2C(C)(F)F)cc1</smiles>

3e $88 \%, 79 \%$ ee 3f $80 \%, 73 \%$ ee 3g $80 \%, 74 \%$ ee<smiles>CN(C)C(=O)N1C=Cc2ccccc2[C@H]1c1ccc2c(c1)OCO2</smiles>

3h $89 \%, 73 \%$ ee<smiles>O=C(N1CCOCC1)N1C=Cc2ccccc2[C@H]1c1ccc(Cl)cc1</smiles>

3i $84 \%, 75 \%$ ee<smiles>CC(=O)N1C=Cc2ccccc2[C@H]1c1ccc(Cl)c(Cl)c1</smiles>

3j $89 \%, 78 \%$ ee<smiles>COc1cc2c(cc1OC)[C@@H](c1ccccc1)N(C(=O)[O-])C=C2</smiles>

3k $93 \%, 60 \%$ ee<smiles>CN(C(=O)[O-])C(=O)N1C=Cc2ccccc2C1c1ccc(F)cc1</smiles>

31 $95 \%, 75 \%$ ee

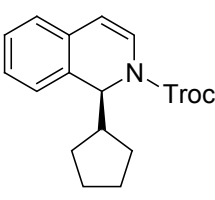

$3 m 56 \%, 77 \%$ ee
${ }^{a}$ Isolated yields based on the isoquinolines;

${ }^{b}$ Determined by chiral HPLC

图 3 异喹啉不对称转移氢化反应的底物范围考察 Figure 3 Isoquinoline substrate scope of asymmetric transfer hydrogenation 


\section{3 结论}

本文设计了一种手性负离子置换策略, 利用氯甲酸 三氯乙酯作为底物活化试剂, 实现了手性磷酸催化的异 喹啉化合物的不对称转移氢化, 反应以优异的分离收率 得到手性 1-取代-1,2-二氢异喹啉, 对映体过量值最高为 $79 \%$. 目前, 我们研究组正在尝试将这种手性负离子置 换策略应用在其它一些不对称反应中.

\section{4 实验部分}

将异喹啉底物 $(0.10 \mathrm{mmol}) 、$ 手性磷酸 $4 \mathbf{a}$ (3.5 mg, 5.0 $\mathrm{mol} \%$ )和碳酸钠 $(16.0 \mathrm{mg}, 0.15 \mathrm{mmol})$ 加入反应瓶中, 加 入 $2.0 \mathrm{~mL}$ 环己烷, 在搅拌下滴加氯甲酸三氯乙酯 $(25.0$ $\mathrm{mg}, 0.12 \mathrm{mmol}$ )的环己烷溶液, 所得反应混合物在室温 下搅拌 $30 \mathrm{~min}$ 至 $1 \mathrm{~h}$. 然后加入 Hantzsch 酯 $\mathbf{5 e}$ (38.0 mg, $0.15 \mathrm{mmol})$. 反应在室温下进行, TLC 检测反应进行程 度, $24 \mathrm{~h}$ 后, 反应完全. 直接采用硅胶柱分离提纯, 流动 相为石油醚：二氯甲烷 $=1 ： 1$ (体积比).

\section{References}

[1] Akiyama, T.; Itoh, J.; Yokota, K.; Fuchibe, K. Angew. Chem. Int. Ed. 2004, 43, 1566.

[2] Uraguchi, D.; Terada, M. J. Am. Chem. Soc. 2004, 126, 5356.

[3] Rueping, M.; Sugiono, E.; Azap, C. Angew. Chem. Int. Ed. 2006,

[4] Itoh, J.; Fuchibe, K.; Akiyama, T. Angew. Chem. Int. Ed. 2006, 45, 4796.

[5] Rueping, M.; Azap, C. Angew. Chem. Int. Ed. 2006, 45, 7832.

[6] Liu, H.; Cun, L.-F.; Mi, A.-Q.; Jiang, Y.-Z.; Gong, L.-Z. Org. Lett. 2006, 8,6023 .

[7] Lv, J.; Zhong, X.-R.; Cheng, J.-P.; Luo, S.-Z. Acta Chim. Sinica 2012, 70, 1518. (吕健, 钟兴仁, 程津培, 罗三中, 化学学报, 2012, $70,1518$.

[8] Huang, J.-Z.; Luo, S.-W.; Gong, L.-Z. Acta Chim. Sinica 2013, 71, 879. (黄建洲, 罗时玮, 龚流柱, 化学学报, 2013, 71, 879.)

[9] Rueping, M.; Sugiono, E.; Azap, C.; Theissmann, T.; Bolte, M. Org. Lett. 2005, 7, 3781 .

[10] Hoffmann, S.; Seayad, A. M.; List, B. Angew. Chem. Int. Ed. 2005, 44, 7424.

[11] Rueping, M.; Antonchick, A. P.; Theissmann, T. Angew. Chem. Int. Ed. 2006, 45, 6751.

[12] Li, G.-L.; Liang, Y.-X.; Antilla, J. C. J. Am. Chem. Soc. 2007, 129, 5830.

[13] Rueping, M.; Sugiono, E.; Schoepke, F.-R. Synlett 2010, 852.

[14] Kang, Q.; Zhao, Z.-A.; You, S.-L. Org. Lett. 2008, 10, 2031.

[15] Wang, D.; Hou, C.-J.; Chen, L.-F.; Liu, X.-N.; An, Q.-D.; Hu, X.-P. Chin. J. Org. Chem. 2013, 33, 1355. (王东, 侯传金, 陈丽风, 刘小 宁, 安庆大, 胡向平, 有机化学, 2013, 33, 1355.)

[16] Jia, Y.-X.; Zhong, J.; Zhu, S.-F.; Zhang, C.-M.; Zhou, Q.-L. Angew. Chem. Int. Ed. 2007, 46, 5565.

[17] Kang, Q.; Zhao, Z.-A.; You, S.-L. J. Am. Chem. Soc. 2007, 129, 1484.

[18] Sheng, Y.-F.; Zhang, A.-J.; Zheng, X.-J.; You, S.-L. Chin. J. Org. Chem. 2008, 28, 605. (盛益飞, 张安将, 郑晓建, 游书力, 有机化 学, 2008, 28, 605.)
[19] Terada, M.; Sorimachi, K. J. Am. Chem. Soc. 2007, 129, 292.

[20] He, Z.-R.; Huang, Y.-Y.; Verpoort, F. Acta Chim. Sinica 2013, 71, 700. (何展荣, 黄毅勇, Verpoort Francis, 化学学报, 2013, 71, 700.)

[21] Xu, S.; Wang, Z.; Zhang, X.; Zhang, X.; Ding, K. Angew. Chem. Int. Ed. 2008, 47, 2840 .

[22] Su, Y.-J.; Shi, F.-Q. Chin. J. Org. Chem. 2010, 30, 486. (苏亚军，史 福强, 有机化学, 2010, 30, 486.)

[23] Wu, X.; Li, M.-L.; Gong, L.-Z. Acta Chim. Sinica 2013, 71, 1091. (吴祥, 李明丽, 龚流柱, 化学学报, 2013, 71, 1091.)

[24] Lv, J.; Luo, S.-Z. Chem. Commun. 2013, 49, 847.

[25] Xu, B.; Zhu, S.-F.; Zhang, Z.-C.; Yu, Z.-X.; Ma, Y.; Zhou, Q.-L. Chem. Sci. 2014, 5, 1442.

[26] Qiu, H.; Li, M.; Jiang, L.-Q.; Lv, F.-P.; Zan, L.; Zhai, C.-W.; Doyle, M. P.; Hu, W.-H. Nature Chemistry 2012, 4, 733.

[27] Qiu, H.; Zhang, D.; Liu, S.-Y.; Qiu, L.; Zhou, J.; Qian, Y.; Zhai, C.-W.; Hu, W.-H. Acta Chim. Sinica 2012, 70, 2484. (邱晃, 张丹, 刘顺英, 邱林, 周俊, 钱宇, 翟昌伟, 胡文浩, 化学学报, 2012, 70, 2484.)

[28] Mahlau, M.; List, B. Angew. Chem. Int. Ed. 2013, 52, 518.

[29] Rauniyar, V.; Lackner, A. D.; Hamilton, G. L.; Toste, F. D. Science 2011, 334, 1681 .

[30] Wang, Y.-M.; Wu, J.; Hoong, C.; Rauniyar, V.; Toste, F. D. J. Am Chem. Soc. 2012, 134, 12928.

[31] Phipps, R. J.; Toste, F. D. J. Am. Chem. Soc. 2013, 135, 1268.

[32] Lackner, A. D.; Samant, A. V.; Toste, F. D. J. Am. Chem. Soc. 2013, $135,14090$.

[33] Zheng, C.; You, S.-L. Chem. Soc. Rev. 2012, 41, 2498.

[34] Rueping, M.; Antonchick, A. P.; Theissmann, T. Angew. Chem. Int Ed. 2006, 45, 3683 .

[35] Glorius, F. Org. Biomol. Chem. 2005, 3, 4171.

[36] Lu, S.-M.; Han, X.-W.; Zhou, Y.-G. Chin. J. Org. Chem. 2005, 25, 634. (卢胜梅, 韩秀文, 周永贵, 有机化学, 2005, 25, 634.)

[37] Zhou, Y.-G. Acc. Chem. Res. 2007, 40, 1357.

[38] Kuwano, R. Heterocycles 2008, 76, 909.

[39] Wang, D.-S.; Chen, Q.-A.; Lu, S.-M.; Zhou, Y.-G. Chem. Rev. 2012, $112,2557$.

[40] Xie, J.-H.; Zhou, Q.-L. Acta Chim. Sinica 2012, 70, 1427. (谢建华, 周其林, 化学学报, 2012, 70, 1427.)

[41] He, Y.-M.; Song, F.-T.; Fan, Q.-H. Top. Curr. Chem. 2014, 343, 145.

[42] Wang, W.-B.; Lu, S.-M.; Yang, P.-Y.; Han, X.-W.; Zhou, Y.-G. J. Am. Chem. Soc. 2003, 125, 10536.

[43] Zhou, H.-F.; Li, Z.-W.; Wang, Z.-J.; Wang, T.-L.; Xu, L.-J.; He, Y.-M.; Fan, Q.-H.; Pan, J.; Gu, L.-Q.; Chan, A. S. C. Angew. Chem. Int. Ed. 2008, 47, 8464 .

[44] Wang, T.-L.; Zhuo, L.-G.; Li, Z.-W.; Chen, F.; Ding, Z.-Y.; He, Y.-M.; Fan, Q.-H.; Xiang, J.-F.; Yu, Z.-X.; Chan, A. S. C. J. Am. Chem. Soc. 2011, 133, 9878.

[45] Lei, A.; Chen, M.; He, M.; Zhang, X. Eur. J. Org. Chem. 2006, 4343.

[46] Kuwano, R.; Sato, K.; Kurokawa, T.; Karube, D.; Ito, Y. J. Am. Chem. Soc. 2000, 122, 7614.

[47] Guo, Q.-S.; Du, D. M.; Xu, J.-X. Angew. Chem. Int. Ed. 2008, 47, 759.

[48] Rueping, M.; Tato, F.; Schoepke, F. R. Chem. Eur. J. 2010, 16, 2688.

[49] Rueping, M.; Antonchick, A. P. Angew. Chem. Int. Ed. 2007, 46, 4562.

[50] Lu, S.-M.; Wang, Y.-Q.; Han, X.-W.; Zhou, Y.-G. Angew. Chem. Int. Ed. 2006, 45, 2260 .

[51] Shi, L.; Ye, Z.-S.; Cao, L.-L.; Guo, R.-N.; Hu, Y.; Zhou, Y.-G. Angew. Chem. Int. Ed. 2012, 51, 8286.

[52] Ye, Z.-S.; Guo, R.-N.; Cai, X.-F.; Chen, M.-W.; Shi, L.; Zhou, Y.-G. Angew. Chem. Int. Ed. 2013, 52, 3685.

[53] Iimuro, A.; Yamaji, K.; Kandula, S.; Nagano, T.; Kita, Y.; Mashima, K. Angew. Chem. Int. Ed. 2013, 52, 2046.

[54] Guo, R.-N.; Cai, X.-F.; Shi, L.; Ye, Z.-S.; Chen, M.-W.; Zhou, Y.-G. Chem. Commun. 2013, 49, 8537. 